\title{
AVALIAÇÃO DA EFICIÊNCIA BANCÁRIA POR MEIO DA ABORDAGEM DE INTERMEDIAÇÃO: UMA ANÁLISE COMPARATIVA DE INSTITUIÇÕES FINANCEIRAS BRASILEIRAS
}

\author{
Murilo Carneiro* \\ marciadeluca@ufc.br \\ Alexandre Pereira Salgado Junior** \\ asalgado@usp.br \\ Lucas Serrão Macoris** \\ 1smacoris@fearp.usp.br \\ * Centro Universitário Moura Lacerda - Ribeirão Preto, SP / Brasil \\ **Universidade de São Paulo - São Paulo, SP / Brasil
}

http://dx.doi.org/10.1590/1413-2311.07315.55484

Recebido em 09/05/2015

Aprovado em 22/06/2016

Disponibilizado em 31/01/2017

Avaliado pelo sistema "double blind review"

Revista Eletrônica de Administração

Editora-chefe: Aurora Zen

ISSN 1413-2311 (versão "on line")

Editada pela Escola de Administração da Universidade Federal do Rio Grande do Sul.

Periodicidade: Quadrimestral

Sistema requerido: Adobe Acrobat Reader

\section{RESUMO}

Este estudo avalia, de forma comparativa, por meio da abordagem de intermediação, o grau de eficiência de 99 instituições financeiras que atuaram, em 2013, no mercado financeiro brasileiro. Foi a atividade de intermediação financeira que gerou o surgimento e a consolidação das instituições financeiras por todo o mundo. Portanto, essa foi uma das razões que motivou a escolha da abordagem de intermediação para se avaliar a eficiência das instituições financeiras. Acredita-se que seja relevante avaliar se tais instituições, no Brasil, estão conseguindo ser eficientes em sua atividade mais tradicional, a intermediação financeira. É importante ressaltar que, além da abordagem de intermediação, há também duas outras principais abordagens para se avaliar a eficiência das instituições financeiras: produção e rentabilidade. A técnica data envelopment analysis (DEA), cuja aplicabilidade para avaliação da eficiência bancária é muito difundida na literatura, foi adotada para calcular os escores de eficiência das instituições, que foram classificadas de acordo com quatro critérios: origem do capital, porte, risco (rating de crédito) e setor de atuação. Foram identificadas 22 instituições com maior grau de eficiência, havendo certa heterogeneidade em relação à origem do capital, porte e setor de atuação. Por outro lado, foi identificado um indício de que há uma relação diretamente proporcional positiva entre o rating de crédito e a eficiência. 
Palavras-chave: Eficiência Bancária; Abordagem de intermediação; Instituições financeiras brasileiras; DEA.

\title{
EVALUATION OF BANKING EFFICIENCY THROUGH INTERMEDIATION APPROACH: A COMPARATIVE ANALYSIS OF BRAZILIAN FINANCIAL INSTITUTIONS
}

\begin{abstract}
This study evaluates, comparatively, through the intermediation approach, the degree of efficiency of 99 financial institutions that acted, in 2013, in the Brazilian financial market. It was the financial intermediation activity that generated the emergence and consolidation of financial institutions around the world. So this was one of the reasons that motivated the choice of intermediation approach to assess the efficiency of financial institutions. It is believed to be relevant to assess whether such institutions in Brazil are getting to be more efficient in their traditional activity, financial intermediation. Importantly, in addition to the intermediation approach, there are also two other major approaches to evaluate the efficiency of financial institutions: production and profitability. The technical data envelopment analysis (DEA), whose applicability to evaluation of bank efficiency is widespread in literature, was adopted to calculate the efficiency scores of institutions, which were classified according to four criteria: the origin of capital, size, risk (credit rating) and sector of activity. 22 institutions with higher efficiency have been identified, with some heterogeneity in relation to the origin of capital, size and sector of activity. On the other hand, an indication that there is a directly proportional relationship between the positive credit rating and efficiency was identified.
\end{abstract}

Keywords: Banking efficiency; intermediation approach; Brazilian financial institutions; DEA.

\section{EVALUACIÓN DE LA EFICIENCIA BANCARIA A TRAVÉS DEL ENFOQUE DE INTERMEDIACIÓN: UN ANÁLISIS COMPARATIVO DE LAS INSTITUCIONES FINANCIERAS BRASILEÑAS}

\begin{abstract}
RESUMEN
Este estudio evalúa, comparativamente, através del enfoque de la intermediación, el grado de eficiencia de 99 instituciones financieras que actuó, en 2013, en el mercado financiero brasileño. Fue la actividad de intermediación financiera que ha generado el surgimiento y consolidación de las instituciones financieras de todo el mundo. Así que esta fue una de las razones que motivaron la elección del enfoque de intermediación para evaluar la eficiencia de las instituciones financieras. Se cree que es pertinente para evaluar si tales instituciones en Brasil están llegando a ser más eficientes en su actividad tradicional, la intermediación financiera. Es importante destacar que, además del enfoque de intermediación , también hay otros dos enfoques principales para evaluar la eficiencia de las instituciones financieras : la
\end{abstract}


producción y la rentabilidad. El técnica data envelopment analysis (DEA), cuya aplicabilidad a la evaluación de la eficiencia bancaria está muy extendida en la literatura, fue adoptado para el cálculo de los índices de eficiencia de las instituciones, que fueron clasificados de acuerdo a cuatro criterios: el origen del capital, el tamaño, el riesgo (calificación crediticia) y sector de actividad. 22 instituciones con mayor eficiencia han sido identificados, con alguna heterogeneidad en relación con el origen del capital, el tamaño y sector de actividad. Por otro lado, una indicación de que existe una relación directamente proporcional entre la calificación de crédito positivo y la eficiencia fue identificado.

Palabras clave: Eficiencia bancaria; enfoque de intermediación; Instituciones financieras brasileñas; DEA.

\section{INTRODUÇÃO}

O setor financeiro e, em especial, o setor bancário exercem um papel fundamental para o desenvolvimento econômico de qualquer país, e não é diferente no Brasil. Segundo Portugal (2011), a existência de um sistema financeiro sólido, ético e eficiente é condição essencial para o desenvolvimento econômico e social sustentável do Brasil. Diante de tais constatações, ressalta-se a relevância deste trabalho, que visa avaliar a eficiência bancária, pois, sem instituições financeiras eficientes, torna-se muito difícil alavancar o crescimento econômico do país.

Desde a Idade Média, quando há o declínio do feudalismo e a ascensão do capitalismo, as instituições financeiras contribuem para o crescimento e consolidação dos países, principalmente, ao captarem recursos financeiros dos agentes econômicos que possuem superávit (ofertadores) e repassarem àqueles que estão em situação de déficit (tomadores). Dessa forma, ao oferecer crédito para capital de giro e investimentos para pessoas físicas e jurídicas, as instituições financeiras fomentam o crescimento das economias. Foi essa atividade, a intermediação financeira, que gerou o surgimento e a consolidação das instituições financeiras por todo o mundo.

Essa foi uma das razões que motivou a escolha da abordagem de intermediação para se avaliar a eficiência das instituições financeiras. Acredita-se que seja relevante avaliar se tais instituições, no Brasil, estão conseguindo ser eficientes em sua atividade mais tradicional, a intermediação financeira. É importante ressaltar que, segundo Macoris; Salgado e Falsarella Júnior (2015), além da abordagem de intermediação, há também duas outras principais abordagens para se avaliar a eficiência das instituições financeiras: produção e rentabilidade.

A escolha também está amparada no estudo elaborado por Berger e Humphrey (1997), os quais constaram que a abordagem de intermediação foi a mais utilizada, nos artigos 
que analisaram, para se mensurar a eficiência das instituições financeiras, apresentando uma frequência de 41\%. Na sequência, foram identificadas frequências de $28 \%$ e $21 \%$, respectivamente, em artigos que utilizaram as abordagens de produção e rentabilidade. As outras abordagens, consideradas de menor importância relativa, representaram apenas $10 \%$. Levando em conta tal estudo, pode-se constatar que a abordagem de intermediação é a mais utilizada para se mensurar a eficiência das instituições financeiras.

Dentre os métodos existentes para se mensurar a eficiência das organizações, será utilizado, neste trabalho, o data envelopment analysis (DEA), conhecido, na língua portuguesa, como análise envoltória de dados. Os dados necessários serão obtidos no site do Banco Central do Brasil, referentes às instituições financeiras que operaram no Brasil, em 2013.

Como, neste estudo, será utilizada a técnica DEA para mensurar a eficiência das instituições financeiras brasileiras, torna-se importante ressaltar que, segundo Macoris; Salgado e Falsarella Júnior (2015), este é um dos principais setores de atividade da economia onde tal técnica tem sido empregada, podendo-se citar também os setores da educação e de políticas públicas.

Para ratificar esta afirmação, pode-se recorrer ao estudo elaborado por Fethi e Pasiouras (2010), onde foi constatado que, dentre 191 trabalhos acadêmicos revisados, publicados em diversos países, 151 deles utilizaram alguma das variações do DEA para estimar as medidas de eficiência operacional dos bancos.

Como as instituições financeiras utilizadas na amostra apresentam características diferentes, serão classificadas levando-se em conta quatro critérios: origem do capital, porte, risco (rating de crédito) e setor de atuação. Este procedimento também foi adotado com o objetivo de se constatar ou refutar a hipótese de que as instituições financeiras podem ter um grau maior ou menor de eficiência, de acordo com seu critério de classificação.

O trabalho está organizado em três blocos. O primeiro, composto pelos três primeiros itens, apresentará conceitos sobre o funcionamento do mercado financeiro e as instituições que o compõem. Na sequência, serão apresentadas considerações sobre as três principais abordagens utilizadas para mensurar a eficiência no setor bancário e, ao final, o DEA será caracterizado como uma das técnicas mais utilizadas para se mensurar a eficiência das organizações.

O segundo bloco, representado pelo quarto item, é destinado à apresentação dos aspectos metodológicos do trabalho. Primeiramente, são definidos alguns aspectos técnicos da pesquisa, para, posteriormente, serem apresentadas as características do estudo elaborado, REAd | Porto Alegre - Edição 85 - N 3 - Setembro / Dezembro 2016 - p. 336 - 359 
ressaltando-se a forma como o universo de pesquisa foi definido, assim como o processo de coleta de dados. O último bloco, representado pelo quinto item, apresentará os resultados obtidos com o estudo, que visa avaliar, em 2013, por meio da abordagem de intermediação e utilizando-se a técnica DEA, quais foram, de forma comparativa, as instituições financeiras brasileiras mais eficientes.

\section{MERCADO FINANCEIRO E INSTITUIÇÕES FINANCEIRAS}

Ao se recorrer ao dicionário, são encontradas diversas definições para a palavra mercado. Dentre elas, a que melhor se adapta ao sentido utilizado neste estudo é: oferta e procura de bens e/ou serviços e/ou capitais. Obviamente, dependendo do que será ofertado e procurado, os mercados podem ser classificados em diversas categorias, como, por exemplo: mercado imobiliário, onde são comprados e vendidos imóveis, e mercado de veículos, onde são comprados e vendidos carros, caminhões, motos etc.

No mercado financeiro compra-se e vende-se dinheiro, e também alguns tipos de papéis, que podem ser facilmente convertidos em dinheiro neste mesmo mercado. Um dos papéis mais conhecidos são as ações, que são ofertadas pelas sociedades anônimas de capital aberto e compradas por investidores. Segundo Rudge e Cavalcante (1993), o mercado financeiro é composto por três participantes:

Tomadores finais: são aqueles que se encontram em posição de déficit financeiro, isto é, aqueles que pretendem gastar (em consumo e/ou investimento) mais do que sua renda. Eles precisam do complemento de poupanças de outros para executar seus planos, dispondose a pagar juros pelo capital que conseguirem;

Ofertadores finais: são aqueles que se encontram em posição de superávit financeiro, isto é, aqueles que pretendem gastar (em consumo e/ou investimento) menos do que sua renda;

Sistema financeiro: é o conjunto de instituições e instrumentos financeiros que possibilitam a transferência de recursos dos ofertadores finais para os tomadores finais, e criam condições para que os papéis tenham liquidez no mercado financeiro.

A existência do sistema financeiro é imprescindível para o funcionamento do mercado financeiro, pois, do contrário, os ofertadores e os tomadores não conseguiriam realizar diretamente suas transações de compra e venda de dinheiro e de papéis. Além disso, também é imprescindível que existam instrumentos financeiros, ou seja, regras e procedimentos para que tais transações sejam realizadas com segurança e transparência. REAd | Porto Alegre - Edição 85 - N 3 - Setembro / Dezembro 2016 - p. 336 - 359 
Didaticamente, o sistema financeiro nacional pode ser subdividido em três grandes subsistemas: normativo, apoio e intermediação financeira.

Subsistema normativo: para que qualquer mercado possa funcionar, de forma organizada e transparente, é imprescindível que existam regras. Além disso, também deve existir quem verifique se tais regras estão sendo cumpridas. Portanto, o subsistema normativo é composto por instituições que definem e controlam as normas de funcionamento do mercado financeiro, tais como: Conselho Monetário Nacional (CMN), Banco Central (BACEN) e Comissão de Valores Mobiliários (CVM);

Subsistema de apoio: enquanto Assaf Neto (2005) classifica como "especiais" as instituições do sistema financeiro que fazem parte desse grupo: Banco do Brasil (BB), Banco Nacional de Desenvolvimento Econômico e Social (BNDES) e Caixa Econômica Federal (CEF), Fortuna (1999) as chama de autoridades de apoio. Neste estudo, foi adotada tal classificação por se acreditar ser mais adequada, pois um dos principais objetivos dessas instituições é apoiar o Governo Federal a colocar em prática algumas de suas políticas. O BB apoia o Governo Federal a colocar em prática sua política oficial de crédito rural (empréstimos a agricultores). O BNDES é responsável pela política de investimentos de longo prazo para aquisição de máquinas e equipamentos, exportação etc. A CEF é o principal agente do Sistema Financeiro de Habitação (SFH), atuando no financiamento da casa própria, principalmente no segmento de baixa renda. É importante ressaltar que o BB e a CEF, além de exercerem funções de apoio ao Governo Federal, também atuam como instituições financeiras bancárias;

Subsistema de intermediação: é composto por instituições que atuam em operações de intermediação financeira; por isso, também é conhecido como subsistema operativo. Didaticamente, de acordo com características semelhantes que possuem, as instituições que compõem o subsistema de intermediação podem ser classificadas em quatro categorias:

$1^{\text {a) }}$ Instituições financeiras bancárias: possuem como principal característica a possibilidade de abrirem contas correntes para seus clientes. Ao movimentarem tais contas, os clientes podem manter um saldo positivo, que é chamado de depósito à vista. As instituições financeiras bancárias podem emprestar para outros clientes, descontando-se o percentual do depósito compulsório, o montante que possuem em depósitos à vista. Nesta categoria são classificadas três tipos de instituições: bancos comerciais, bancos múltiplos e caixas econômicas;

$2^{\mathrm{a})}$ Instituições financeiras não bancárias: ao contrário das instituições financeiras bancárias, as instituições classificadas nesta categoria não possuem permissão para abrir REAd | Porto Alegre - Edição 85 - № 3 - Setembro / Dezembro 2016 - p. 336 - 359 
contas correntes para seus clientes, ficando impossibilitadas de utilizarem recursos provenientes de depósitos à vista. Nesta categoria estão inseridos os bancos de investimentos, as sociedades de crédito, financiamento e investimento (mais conhecidas pelo nome de financeiras) e as sociedades de arrendamento mercantil;

$3^{a}$ ) Instituições auxiliares: são instituições que auxiliam e viabilizam a existência do mercado de capitais, que é uma subdivisão do mercado financeiro onde são negociados os valores mobiliários (debêntures, commercial papers e ações). Nesta categoria estão inseridas as corretoras de valores e a bolsa de valores;

$4^{\mathrm{a}}$ ) Instituições não financeiras: são instituições que, apesar de não serem consideradas instituições financeiras, exercem papel importante no mercado financeiro. Nesta categoria, estão inseridas as sociedades de fomento comercial - factorings, as companhias seguradoras e as administradoras de cartão de crédito.

\section{ABORDAGENS PARA MENSURAR A EFICIÊNCIA NO SETOR BANCÁRIO}

Segundo Carneiro (2012), a administração pode ser definida como um processo composto por quatro etapas interdependentes e cíclicas (planejamento, organização, direção e controle), que visa levar as organizações a utilizarem seus recursos da melhor maneira possível, tornando-as eficientes, para que consigam atingir seus objetivos, tornando-as eficazes.

Obviamente, quanto mais eficientes forem as organizações, ou seja, quanto melhor utilizarem os recursos que têm disponíveis, maiores as chances de se tornarem eficazes, ou seja, maiores as chances de atingirem seus objetivos. Portanto, para se avaliar a eficiência de uma organização, deve-se avaliar se os recursos que possui estão sendo utilizados da melhor maneira possível.

De acordo com o enfoque sistêmico, eficácia significa a capacidade de realizar um objetivo ou resolver um problema. Quanto maior o acerto na realização do objetivo ou resolução do problema, mais elevado é o grau de eficácia da organização. Portanto, segundo Maximiano (2011), a eficácia deve ser avaliada comparando-se os objetivos que se pretendia alcançar com os resultados efetivamente alcançados. Em relação à eficiência, pode-se dizer que um sistema eficiente é aquele que utiliza racionalmente seus recursos. Quanto mais racional for a utilização dos recursos, mais produtivo e eficiente será o sistema. De forma geral, Maximiano (2011) afirma que a eficiência deve ser avaliada comparando-se os resultados alcançados com os recursos utilizados.

REAd | Porto Alegre - Edição 85 - №3 - Setembro / Dezembro 2016 - p. 336 - 359 
Como este trabalho tem como objetivo avaliar a eficiência do setor bancário, torna-se imprescindível apresentar as abordagens que têm sido utilizadas pelos pesquisadores visando tal intuito. É importante ressaltar que, segundo Liu (2010), a eficiência bancária tem sido um horizonte de pesquisa que tem atraído não somente acadêmicos, mas também os tomadores de decisão de tais organizações.

Após a elaboração de uma revisão bibliográfica sobre como deve ser desenvolvida a avaliação da eficiência do setor bancário, constatou-se que os pesquisadores sugerem uma análise entre inputs e outputs. De forma simplificada, pode-se dizer que os inputs são os recursos que estão sendo utilizados pela organização em sua atividade, enquanto os outputs são os resultados obtidos com a utilização de tais recursos.

Mester (1997) afirma que uma instituição financeira pode ser considerada ineficiente se utiliza um nível alto de inputs e obtém um nível baixo de outputs. Esta ideia é ratificada por Hasan; Kamil; Mustafa e Baten (2012), ao concluírem que uma instituição financeira eficiente é aquela que, dado um determinado nível de inputs, obtém resultados máximos de outputs, bem como aquela que minimiza os inputs utilizados na obtenção de determinados níveis de outputs.

Dependendo dos tipos de inputs e outputs que são utilizados em determinado estudo, podem ser definidas diferentes abordagens para se avaliar a eficiência do setor bancário. Yang (2009) comenta que há inúmeras abordagens que podem ser utilizadas, cada uma visando obter um aspecto distinto de eficiência. Portanto, não se pode afirmar que há uma abordagem melhor que outra, pois, de acordo com Ferrier e Lovell (1990), a abordagem mais adequada depende, sobretudo, do foco que está sendo dado ao estudo da eficiência.

Em relação aos tipos de abordagens que podem ser utilizadas para avaliar a eficiência do setor bancário, não foi encontrado um consenso entre os autores pesquisados. Tulkens e Vanden Eeckaut (1995), por exemplo, listam cinco tipos de abordagens: intermediação, utilidade, produção, rentabilidade e gerenciamento de risco.

Apesar de não haver consenso, a maioria dos autores considera duas abordagens, a de intermediação e a de produção (BERGER E HUMPHREY, 1997; FETHI e PASIOURAS, 2010; LIU, 2010; SATHYE, 2003; TORTOSA-AUSINA, 2002). Outros autores, como, por exemplo, Macoris; Salgado e Falsarella Júnior (2015), citam três tipos de abordagens: produção, intermediação e rentabilidade. Diante de tais considerações, optou-se por apresentar as principais características das três abordagens mais citadas.

\subsection{Abordagem de Intermediação}

REAd | Porto Alegre - Edição 85 - №3 - Setembro / Dezembro 2016 - p. 336 - 359 
A abordagem de intermediação, desenvolvida por Sealey e Lindley (1977), trata os bancos como intermediários financeiros, pois leva em conta que seu principal objetivo é captar recursos dos ofertadores e repassá-los aos tomadores.

Tal abordagem, por exemplo, foi utilizada no estudo elaborado por Staub; Silva e Souza e Tabak (2010), levando em conta que, com tal configuração, os bancos podem ser vistos como intermediadores de recursos financeiros entre ofertadores e tomadores. Em tal estudo, o montante de recursos captados e as despesas de juros foram considerados os principais inputs do processo, contando também com as despesas operacionais (líquidas de despesas com pessoal) e as despesas com pessoal. Dessa forma, os inputs utilizados no estudo contemplaram, conforme proposto por Sealey e Lindley (1977), trabalho, custos materiais incorridos na produção dos serviços para os clientes e capital (fundos captados). Como outputs, foram utilizados, basicamente, empréstimos e investimentos, ou seja, o destino dos recursos financeiros captados pelo banco na atividade de intermediação. Assim, para ser considerado eficiente, um banco deve otimizar seus inputs, como despesas de juros, despesas operacionais e despesas com pessoal, visando maximizar seus outputs, como empréstimos ou investimentos.

Em seu estudo, Liu (2009) também utilizou a abordagem de intermediação, utilizando três tipos de inputs: depósitos, despesas com juros e despesas não relacionadas a juros. Yeh (apud LIU, 2009) comenta que tais variáveis representam os custos relativos ao trabalho, administração, equipamentos e fundos captados para as operações do banco, além de ser a fonte de recursos disponíveis para empréstimos. No estudo citado, foram utilizados como outputs os empréstimos totais, as receitas com juros e as receitas não relacionadas aos juros, representando, segundo Yeh (apud LIU, 2009), a receita do banco e sua atividade de maior rentabilidade.

\subsection{Abordagem de Produção}

Berger e Humphrey (1997) afirmam que a abordagem de produção está entre as principais escolhas para se medir a eficiência do fluxo dos serviços bancários. Tal abordagem define como premissa que os bancos devem visar, como função principal, a disponibilização de serviços aos seus clientes.

Kuussaari (1993) comenta que devem ser levados em conta vários tipos de serviços, não somente serviços de pagamento e empréstimos, tais como: monitoramento e avaliação de REAd | Porto Alegre - Edição 85 - № 3 - Setembro / Dezembro 2016 - p. 336 - 359 
riscos de investimento, cobranças, custódia de títulos, transações com ações e outros valores mobiliários, proteção e vários serviços de aconselhamento. De forma simplificada, pode-se dizer que tal abordagem preocupa-se em mensurar a capacidade que os bancos possuem em prover serviços de diversas naturezas aos seus clientes, levando em consideração os insumos materiais que possui.

Normalmente, os inputs utilizados para se avaliar a eficiência dos bancos, levando em conta tal abordagem, são relacionados ao trabalho, materiais e capital investido em edificações e máquinas, como máquinas para processamento de dados, caixas eletrônicos e redes eletrônicas entre bancos. Dessa forma, como a abordagem de produção leva em conta os serviços físicos, os custos dos serviços bancários são medidos pelos custos operacionais do banco. Os outputs são representados, geralmente, pelo número dos diferentes tipos de empréstimos e contas de depósitos ou pelo valor monetário destes.

\subsection{Abordagem de Rentabilidade}

Além das abordagens de intermediação e de produção, cabe destacar a abordagem de rentabilidade, pois também foram identificados alguns trabalhos que a utilizaram. O estudo da eficiência do setor bancário, por meio da abordagem de rentabilidade, segundo Fethi e Pasiouras (2010), deve ser elaborado utilizando-se como outputs os componentes da receita dos bancos, como receitas com juros e receitas não relacionadas a juros. Como inputs, devem ser utilizados alguns componentes de custo dos bancos, como despesas com pessoal e despesas com juros.

Conclui-se que, por meio da abordagem de rentabilidade, os bancos eficientes são aqueles que conseguem minimizar seus custos e maximizar suas receitas, pois foi constatado que os pesquisadores que adotam tal abordagem consideram como inputs, frequentemente, todos os fatores de custo relacionados à atividade geradora de receita, como custos administrativos, custos de pessoal e custos relacionados à captação de recursos. Em contrapartida, os outputs utilizados estão relacionados ao conjunto de receitas geradas, por meio de serviços e de transações financeiras.

Para ratificar tal conclusão, pode-se utilizar, como exemplo, o estudo de Avkiran (2011), que adotou a abordagem de rentabilidade para avaliar a eficiência do setor bancário. Tal autor levou em conta dois inputs que deveriam ser minimizados: despesas de juros e despesas não relacionadas a juros, assim como dois outputs que deveriam ser maximizados: receitas de juros e receitas não relacionadas aos juros. Partindo do princípio que tais inputs e REAd | Porto Alegre - Edição 85 - N 3 - Setembro / Dezembro 2016 - p. 336 - 359 
outputs estão relacionados aos custos e receitas de um banco, servem, consequentemente, para avaliar sua eficiência em obter receitas a partir de uma base de custos incorrida no processo de geração de tais receitas.

\section{O DEA COMO TÉCNICA PARA MENSURAR A EFICIÊNCIA DAS ORGANIZAÇÕES}

Entre as técnicas existentes para mensurar a eficiência das organizações, Cooper; Seiford e Zhu (2011) sugerem a utilização do data envelopment analysis (DEA), conhecido, na língua portuguesa, como análise envoltória de dados. Tais autores comentam que, a partir de um artigo elaborado por Charnes, Cooper e Rhodes, publicado no European Journal of Operational Research, em 1978, o DEA começou a ser difundido no meio acadêmico e, de forma progressiva, passou a ser aceito pela comunidade científica como uma ferramenta de modelagem dos processos operacionais das organizações. Para ratificar tal afirmação, pode-se recorrer a Tavares (2002), pois tal autor identificou, de 1978 a 2001, 3183 publicações que utilizaram o DEA.

O DEA é considerado uma técnica não paramétrica, pois não depende de inferências estatísticas para a resolução. Esta característica gera, em relação a outras técnicas, uma vantagem operacional ao DEA, visto que não se torna necessário elaborar aproximações a modelos já conhecidos, como linear, logarítmico, exponencial etc. O DEA também é considerado uma técnica multivariável, pois busca mensurar a eficiência de uma certa unidade de produção, de forma comparativa, em relação às demais de um determinado grupo.

A técnica DEA assume o princípio de que as unidades de produção possuem poder de decisão; portanto, podem interferir na produtividade delas mesmas. Para tanto, as unidades de produção podem organizar os recursos (inputs) que têm disponíveis da forma que acharem mais conveniente, visando atingir resultados (outputs) mais satisfatórios. É por este motivo que Charnes; Cooper e Rhodes (1978) chamam as unidades de produção de decision making units (DMU), na língua portuguesa: unidades de tomada de decisão.

Desde seu surgimento, a técnica DEA tem sido amplamente utilizada e ampliada, pois foram criadas diversas outras variações derivadas da técnica original. Contudo, as modelagens para o DEA mais utilizadas são o CCR (recebeu essa denominação devido às iniciais dos autores que, em 1978, a desenvolveram: Charnes, Cooper e Rhodes) e BCC (recebeu essa denominação devido às iniciais dos autores que, em 1984, a desenvolveram: Banker, Charnes e Cooper).

REAd | Porto Alegre - Edição 85 - N 3 - Setembro / Dezembro 2016 - p. 336 - 359 
Os casos tratados por Charnes; Cooper e Rhodes (1978) assumiram a premissa, contestada, posteriormente, por Banker; Charnes e Cooper (1984), de que as DMUs sempre atuam em ambientes de retornos constantes de escala, ou seja, não há ganhos nem perdas de escala nos ambientes de negócios nos quais as DMUs estão inseridas. É por esse motivo que a modelagem CCR também passou a ser conhecida pela sigla CRS (Constant returns to scale), na língua portuguesa: retornos constantes de escala. Por outro lado, a modelagem para o DEA proposta por Banker; Charnes e Cooper (1984) assume a premissa de que as DMUs atuam em ambientes onde há ganhos e perdas de escala. É por esse motivo que a modelagem BCC também passou a ser conhecida pela sigla VRS (variable returns to scale), na língua portuguesa: retornos variáveis de escala.

A ideia central em se mensurar a eficiência das organizações por meio da técnica DEA é identificar, matematicamente, em um conjunto de DMUs, as que possuem as maiores produtividades relativas, sendo consideradas, portanto, mais eficientes que as demais. A partir dessas DMUs, consideradas mais eficientes, também é calculada a folga de ineficiência que as outras DMUs, consideradas menos eficientes, possuem.

O grupo das DMUs com as maiores produtividades relativas identificadas formará a chamada fronteira de eficiência. Tais DMUs delimitarão o conjunto possível de produção, na língua inglesa: production possibility set (PPS). Pode-se dizer que, na prática, o PPS representa, comparativamente, o nível ideal de produtividade das DMUs com os recursos (inputs) que estão sendo utilizados.

Outra característica importante da técnica DEA, segundo Falsarela Júnior (2014), é a possibilidade de ser feita uma ponderação entre os inputs e outputs das DMUs, utilizando-se pesos livres ou previamente definidos, por meio de uma programação linear.

\section{ASPECTOS METODOLÓGICOS}

A apresentação dos aspectos metodológicos do trabalho foi dividida em duas partes. Primeiramente, são definidos alguns aspectos técnicos da pesquisa, para, posteriormente, serem apresentadas as características do estudo elaborado, ressaltando-se a forma como o universo de pesquisa foi definido, assim como o processo de coleta de dados.

\subsection{Caracterização da pesquisa}


Uma revisão da literatura que aborda metodologia científica levou à conclusão que devem ser definidos quatro tipos de aspectos: tipo de pesquisa, método de abordagem, método de procedimento e tipo de abordagem.

Para a elaboração do estudo proposto, adotou-se a pesquisa aplicada como tipo de pesquisa, e o método dedutivo como método de abordagem. Após a definição desses dois aspectos, devem ser definidos o método de procedimento de pesquisa e o tipo de abordagem. Neste estudo, foram adotadas a análise de documentos como método de procedimento de pesquisa e a abordagem quantitativa como tipo de abordagem. Na sequência, são caracterizados os aspectos metodológicos utilizados em pesquisas acadêmicas, assim como considerações que justificam os que foram adotados.

O primeiro aspecto a ser definido em uma pesquisa acadêmica é o tipo de pesquisa. De acordo com Andrade (2004), os tipos de pesquisas podem ser classificados de várias formas, segundo critérios diversos, que adotam diferentes pontos de vista. Para Salomon (2001), existem três tipos de pesquisa: exploratórias e descritivas, aplicadas e puras ou teóricas.

Ao se observar as características dos três tipos de pesquisa, verifica-se que a pesquisa aplicada é a que melhor se adapta ao objetivo do estudo proposto, que é analisar a eficiência das instituições financeiras brasileiras por meio da abordagem de intermediação.

Após a definição do tipo, é necessário que se defina o método de pesquisa. Para Lakatos e Marconi (1995), método de pesquisa é o conjunto das atividades sistemáticas e racionais que permite alcançar o objetivo. De acordo com tais autoras, os métodos de pesquisa podem ser divididos em duas classes: métodos de abordagem e métodos de procedimento. Enquanto os métodos de abordagem tratam de questões de forma mais ampla e genérica, os métodos de procedimento são etapas mais concretas de busca de solução. Os métodos de abordagem são classificados em quatro tipos: indutivo, dedutivo, hipotético-dedutivo e dialético.

O método de abordagem adotado neste estudo é o dedutivo, pois, utilizando-se como subsídio o referencial teórico existente sobre o processo de análise da eficiência bancária por meio da abordagem de intermediação, o objetivo da pesquisa será avaliar a eficiência das instituições financeiras brasileiras.

Em relação aos métodos de procedimento de pesquisa, dentre as diversas tipologias desenvolvidas pelos autores para classificar os métodos de procedimento de pesquisa, apresenta-se a tipologia de Salomon (2001), pois se acredita que seja mais sintética e didática. O autor classifica os métodos de procedimento de pesquisa em três tipos: histórica, descritiva REAd | Porto Alegre - Edição 85 - N 3 - Setembro / Dezembro 2016 - p. 336 - 359 
e experimental. Ressalta-se que, dentre as modalidades existentes para se elaborar a pesquisa descritiva, destacam-se: estudos de conjuntos (surveys), estudos de caso e análise de documentos.

Dentre os métodos de procedimento de pesquisa apresentados, o que mais se adapta ao objetivo deste trabalho é a pesquisa descritiva, na modalidade análise de documentos. Essa escolha está amparada nas observações de Yin (2004) sobre as características de tal método. De acordo com tal autor, a pesquisa descritiva investiga fenômenos contemporâneos dentro do contexto da vida real, quando as fronteiras entre fenômeno e contexto não são muito claras. Acredita-se que este seja o caso da análise da eficiência de instituições financeiras, pelo fato de tal processo ainda não se encontrar consolidado na literatura acadêmica brasileira, principalmente pela escassez de estudos abordando instituições que atuam no Brasil.

A última etapa na definição dos aspectos metodológicos deve ser a escolha do tipo de abordagem. Existem dois tipos: a quantitativa e a qualitativa. Neste estudo, utilizar-se-á a abordagem quantitativa, visto que seu objetivo será analisar, por meio de inferências estatísticas e correlações, a eficiência das instituições financeiras brasileiras pela ótica da abordagem de intermediação.

\subsection{Universo da pesquisa e coleta de dados}

É importante destacar que, para que os resultados obtidos utilizando-se a técnica DEA sejam efetivamente relevantes para se mensurar, comparativamente, a eficiência das organizações, é imprescindível que as variáveis de input e output a serem utilizadas no modelo possuam, dentro do ambiente de negócios no qual elas atuam, grande relevância. A preocupação com uma seleção criteriosa de variáveis para o sucesso do modelo é compartilhada por diversos autores (FERREIRA e GOMES, 2012; SENRA, NANCI, MELLO e MEZA, 2007; THANASSOULIS, 2001).

Para Ferreira e Gomes (2012), o processo de seleção das variáveis que serão utilizadas deve levar em conta um profundo conhecimento do segmento de atividade onde atuam as organizações que serão analisadas. Tal autor comenta que, visando tal intuito, podese recorrer a especialistas que conheçam o segmento de atividade, para que forneçam subsídios para se definir os inputs e outputs que serão utilizados.

Neste estudo, para que se pudesse analisar, comparativamente, a eficiência das instituições financeiras brasileiras por meio da abordagem de intermediação, foram definidas seis variáveis (vide quadro 1). Como tal abordagem, segundo Sealey e Lindley (1977), trata os REAd | Porto Alegre - Edição 85 - № 3 - Setembro / Dezembro 2016 - p. 336 - 359 
bancos como intermediários financeiros, pois leva em conta que seu principal objetivo é captar recursos dos ofertadores e repassá-los aos tomadores, acredita-se que os inputs e outputs selecionados representem, eficazmente, uma medida da eficiência dessa atividade. Tais variáveis permitirão que seja mensurado o quanto de capital as instituições conseguiram intermediar e quanto conseguiram obter com receitas financeiras, utilizando os recursos disponíveis e seus respectivos custos, sendo estes recursos o trabalho (número de pessoas), as despesas com juros sobre o capital utilizado e o montante de capital (total de depósitos).

\begin{tabular}{|l|l|}
\multicolumn{2}{|c|}{ Quadro 1 - Inputs e outputs utilizados no estudo } \\
\hline INPUTS & OUTPUTS \\
\hline Total de depósitos & Empréstimos (operações de crédito) \\
\hline Número de pessoas & Receitas de intermediação financeira \\
\hline Despesas com juros (intermediação financeira) & Investimentos/aplicações \\
\hline
\end{tabular}

Fonte: Elaborado pelos autores

Para a definição dessas variáveis, foram levados em conta dois critérios. O primeiro está relacionado aos tipos de inputs e outputs que têm sido utilizados em estudos envolvendo a eficiência do setor bancário por meio da abordagem de intermediação, os quais já foram citados no referencial teórico. Portanto, as variáveis definidas estão alinhadas a diversos outros estudos que abordam o mesmo tema. Para ratificar esta afirmação, pode-se recorrer, por exemplo, ao estudo elaborado por Fethi e Pasiouras (2010), que, ao analisarem diversos trabalhos relacionados à aplicação do DEA em instituições financeiras, encontraram 95 aplicações onde o valor monetário dos depósitos era utilizado como um input.

O segundo critério adotado para a definição das variáveis tem relação com a possibilidade real de as obter. Portanto, a escolha também levou em conta um levantamento das variáveis que estavam disponíveis no Plano Contábil das Instituições do Sistema Financeiro Nacional (COSIF). É importante destacar que os planos de contas utilizados pelas instituições financeiras são definidos em cada país, o que nem sempre permite que as mesmas variáveis possam ser avaliadas para diferentes países. Pode-se citar este fato como uma limitação apresentada por este estudo, pois, ao se elaborar a revisão do referencial teórico, foram identificados estudos internacionais que utilizaram variáveis que não estão disponíveis para as instituições financeiras que operam no Brasil.

Os dados para as variáveis utilizadas neste estudo, que estão expressos na unidade de tempo ano, são referentes às instituições financeiras que operaram no Brasil, em 2013, e foram obtidos no Banco Central do Brasil (2014), na área de informações cadastrais e contábeis. A amostra inicial continha 135 instituições financeiras, porém, após a definição das 
variáveis, algumas precisaram ser excluídas, pelo fato de não apresentarem todos os inputs e outputs necessários. Dessa forma, o estudo foi realizado com uma amostra efetiva de 99 instituições.

Como as instituições financeiras utilizadas na amostra possuem características diferentes, considerou-se extremamente importante classificá-las. Para elaborar tal classificação, foram utilizados quatro critérios: origem do capital, porte, risco (rating de crédito) e setor de atuação.

A informação referente à origem de capital foi obtida no Banco Central, que classifica as instituições financeiras em seis categorias: capital estrangeiro, privado nacional, privado nacional com controle estrangeiro, privado nacional com participação estrangeira, público federal e público estadual.

Em relação aos critérios para tal classificação, é importante comentar que as instituições financeiras, classificadas como capital estrangeiro, são filiais de instituições cuja matriz situa-se em outro país. As classificadas como privado nacional com controle estrangeiro são aquelas na qual a participação estrangeira é maior ou igual a $50 \%$ do capital votante. Por outro lado, privado nacional com participação estrangeira são aquelas instituições cuja participação estrangeira é maior que $10 \%$ e menor que $50 \%$ do capital votante.

As informações referentes aos outros três critérios de classificação foram obtidas por meio do software Visionarium, que, segundo Langkamp (2014), é um dos principais sistemas utilizados para avaliação de risco de crédito corporativo no Brasil. Em relação ao porte, utilizando-se como critério o valor do patrimônio líquido, que é dividido em quartis, são definidas quatro categorias. No primeiro quartil, encontram-se os bancos de porte micro; no segundo, os bancos de pequeno porte; no terceiro, os bancos de médio porte e, no quarto, os bancos de grande porte.

Quanto aos setores de atuação, há oito categorias: Bancos de Desenvolvimento, Corporate, Câmbio, CDC/Consumo, Montadora/Veículos, Pequenas e Médias Empresas, Produtos Serviços e Tesouraria e Varejo. Em relação ao risco (rating de crédito), as instituições financeiras são classificadas em sete categorias: AAA, AA, A, BBB, BB, B e CCC. É importante destacar que as instituições classificadas como AAA apresentam o menor risco de insolvência, enquanto as classificadas como CCC apresentam um risco maior de não honrar seus compromissos financeiros.

Os dados obtidos foram processados por meio do software Frontier®, que utiliza a técnica DEA - BCC, desenvolvida por Banker; Charnes e Cooper (1984), também conhecida como VRS (variable returns to scale). A escolha por tal técnica deve-se ao fato de se REAd | Porto Alegre - Edição 85 - N 3 - Setembro / Dezembro 2016 - p. 336 - 359 
considerar que as instituições financeiras atuam em ambientes onde há ganhos e perdas de escala, uma premissa da modelagem BCC.

\section{APRESENTAÇÃO DOS RESULTADOS}

Ao se utilizar a técnica DEA para mensurar a eficiência, identifica-se, em um conjunto de organizações, as que possuem as maiores produtividades relativas, sendo consideradas, portanto, mais eficientes que as demais. Após o processamento dos dados, foram identificadas 22 instituições com um escore DEA de 100\%, representando, de forma comparativa com as demais, o nível máximo de eficiência. A tabela 1 apresenta as 22 instituições financeiras brasileiras mais eficientes, em 2013, avaliadas por meio da abordagem de intermediação.

Tabela 1 - Instituições financeiras mais eficientes pela abordagem de intermediação

\begin{tabular}{|c|c|c|c|c|}
\hline Instituições Financeiras & Origem do Capital & Setor de Atuação & Porte & Rating \\
\hline $\begin{array}{l}\text { BANCO AZTECA DO } \\
\text { BRASIL S.A. }\end{array}$ & $\begin{array}{l}\text { P. N. com Controle } \\
\text { Estrangeiro }\end{array}$ & CDC/Consumo & Micro & \# \\
\hline ALFA & Privado Nacional (PN) & $\begin{array}{l}\text { Produtos, Serviços e } \\
\text { Tesouraria }\end{array}$ & Grande & AA \\
\hline JP MORGAN CHASE & $\begin{array}{l}\text { P. N. com Controle } \\
\text { Estrangeiro }\end{array}$ & $\begin{array}{l}\text { Produtos, Serviços e } \\
\text { Tesouraria }\end{array}$ & Grande & A \\
\hline $\begin{array}{l}\text { CAIXA ECONOMICA } \\
\text { FEDERAL }\end{array}$ & Público Federal & Varejo & Grande & AAA \\
\hline CREDIT SUISSE & $\begin{array}{l}\text { P. N. com Controle } \\
\text { Estrangeiro }\end{array}$ & $\begin{array}{l}\text { Produtos, Serviços e } \\
\text { Tesouraria }\end{array}$ & Grande & A \\
\hline JOHN DEERE & $\begin{array}{l}\text { P. N. com Controle } \\
\text { Estrangeiro }\end{array}$ & Montadora/Veículos & Médio & $\#$ \\
\hline BCO CARGILL S.A. & $\begin{array}{l}\text { P. N. com Controle } \\
\text { Estrangeiro }\end{array}$ & $\begin{array}{l}\text { Produtos, Serviços e } \\
\text { Tesouraria }\end{array}$ & Pequeno & $\mathrm{B}$ \\
\hline BCO KEB DO BRASIL SA & $\begin{array}{l}\text { P. N. com Controle } \\
\text { Estrangeiro }\end{array}$ & Cambio & Micro & A \\
\hline BANCO DO BRASIL & Público Federal & Varejo & Grande & AAA \\
\hline BNDES & Público Federal & $\begin{array}{l}\text { Banco de } \\
\text { Desenvolvimento }\end{array}$ & Grande & AAA \\
\hline $\begin{array}{l}\text { BCO RABOBANK INTL } \\
\text { BRASIL S.A. }\end{array}$ & $\begin{array}{l}\text { P. N. com Controle } \\
\text { Estrangeiro }\end{array}$ & $\begin{array}{l}\text { Produtos, Serviços e } \\
\text { Tesouraria }\end{array}$ & Médio & AA \\
\hline $\begin{array}{l}\text { BCO LA NACION } \\
\text { ARGENTINA }\end{array}$ & $\begin{array}{l}\text { P. N. com Controle } \\
\text { Estrangeiro }\end{array}$ & Cambio & Micro & \# \\
\hline BCO DES. DE MG S.A. & Público Estadual & $\begin{array}{l}\text { Banco de } \\
\text { Desenvolvimento }\end{array}$ & Grande & \# \\
\hline BCO DES. DO ES S.A. & Público Estadual & $\begin{array}{l}\text { Banco de } \\
\text { Desenvolvimento }\end{array}$ & Pequeno & $\#$ \\
\hline BRADESCO & Privado Nacional (PN) & CDC/Consumo & Grande & AAA \\
\hline BCO VOLVO BRASIL S.A. & $\begin{array}{l}\text { P. N. com Controle } \\
\text { Estrangeiro }\end{array}$ & Montadora/Veículos & Médio & $\#$ \\
\hline ITAU & Privado Nacional (PN) & Varejo & Grande & AAA \\
\hline BTG PACTUAL & $\begin{array}{l}\text { P. N. com Participação } \\
\text { Estrangeira }\end{array}$ & $\begin{array}{l}\text { Produtos, Serviços e } \\
\text { Tesouraria }\end{array}$ & Grande & AA \\
\hline
\end{tabular}




\begin{tabular}{lllll}
\hline SAFRA & Privado Nacional (PN) & Corporate & Grande & AAA \\
\hline BANCO RANDON S.A. & $\begin{array}{l}\text { P. N. com Participação } \\
\text { Estrangeira }\end{array}$ & Montadora/Veículos & Micro & $\#$ \\
\hline $\begin{array}{l}\text { BCO WOORI BANK DO } \\
\text { BRASIL S.A. }\end{array}$ & $\begin{array}{l}\text { P. N. com Controle } \\
\text { Estrangeiro }\end{array}$ & Cambio & Micro & A \\
\hline BANCO BRACCE S.A. & $\begin{array}{l}\text { P. N. com Controle } \\
\text { Estrangeiro }\end{array}$ & CDC/Consumo & Micro & B \\
\hline
\end{tabular}

Fonte: Elaborado pelos autores

No conjunto das instituições financeiras brasileiras mais eficientes, levando-se em conta a origem do capital (tabela 2), destacam-se as instituições financeiras privadas nacionais com participação estrangeira e as público-federais, com escores DEA médios de, respectivamente, $87,66 \%$ e 79,02\%. Em contrapartida, as instituições financeiras privadas nacionais não obtiveram um resultado muito satisfatório, apresentando um escore DEA médio de $34,35 \%$.

Os resultados obtidos vão, em parte, de encontro com os identificados por Staub; Silva e Souza e Tabak (2010), em um estudo elaborado com instituições financeiras brasileiras, utilizando dados dos anos 2000 a 2007. Tais autores também identificaram que as instituições financeiras públicas são mais eficientes que as privadas. Por outro lado, no estudo de Staub; Silva e Souza e Tabak (2010), as instituições financeiras com participação estrangeira se mostraram economicamente ineficientes.

Tabela 2 - Instituições financeiras mais eficientes: análise por origem do capital

\begin{tabular}{lccccc}
\hline Origem do Capital & Eficientes & \% do Total & Amostra & \% do Total & Escore DEA Médio \\
\hline Estrangeiro & 0 & $0,00 \%$ & 2 & $2,02 \%$ & $67,09 \%$ \\
\hline Privado Nacional (P.N.) & 4 & $18,18 \%$ & 41 & $41,41 \%$ & $34,35 \%$ \\
\hline P.N. com Controle Estrangeiro & 11 & $50,00 \%$ & 41 & $41,41 \%$ & $54,47 \%$ \\
\hline P.N. com Participação Estrang. & 2 & $9,09 \%$ & 3 & $3,03 \%$ & $87,66 \%$ \\
\hline Público Estadual & 2 & $9,09 \%$ & 7 & $7,07 \%$ & $43,11 \%$ \\
\hline Público Federal & 3 & $13,64 \%$ & 5 & $5,05 \%$ & $79,02 \%$ \\
\hline Total & $\mathbf{2 2}$ & $\mathbf{1 0 0 , 0 0 \%}$ & $\mathbf{9 9}$ & $\mathbf{1 0 0 , 0 0 \%}$ & $\mathbf{4 7 , 8 4 \%}$ \\
\hline
\end{tabular}

Fonte: Elaborado pelos autores

No conjunto das instituições financeiras brasileiras mais eficientes, levando-se em conta o setor de atuação (tabela 3), destacam-se os bancos de desenvolvimento e as instituições que atuam com operações cambiais, com escores DEA médios de, respectivamente, $100,00 \%$ e $83,27 \%$. As instituições que atuam no setor de pequenas e médias 
empresas, em contrapartida, não obtiveram resultados expressivos, apresentando um escore DEA médio de $23,42 \%$.

Tabela 3 - Instituições financeiras mais eficientes: análise por setor de atuação

\begin{tabular}{lccccc}
\hline Setor de Atuação & Eficientes & \% do Total & Amostra & \% do Total & Escore DEA Médio \\
\hline Varejo & 3 & $13,64 \%$ & 15 & $15,15 \%$ & $44,91 \%$ \\
\hline Produtos, Serviços e Tesouraria & 6 & $27,26 \%$ & 14 & $14,14 \%$ & $65,36 \%$ \\
\hline Pequenas e Médias Empresas & 0 & $0,00 \%$ & 17 & $17,17 \%$ & $23,42 \%$ \\
\hline Montadora/Veículos & 3 & $13,64 \%$ & 13 & $13,13 \%$ & $50,08 \%$ \\
\hline Corporate & 1 & $4,54 \%$ & 14 & $14,14 \%$ & $50,59 \%$ \\
\hline Câmbio & 3 & $13,64 \%$ & 4 & $4,04 \%$ & $83,27 \%$ \\
\hline Banco de Desenvolvimento & 3 & $13,64 \%$ & 3 & $3,03 \%$ & $100,00 \%$ \\
\hline CDC/Consumo & 3 & $13,64 \%$ & 19 & $19,20 \%$ & $43,33 \%$ \\
\hline Total & $\mathbf{2 2}$ & $\mathbf{1 0 0 , 0 0 \%}$ & $\mathbf{9 9}$ & $\mathbf{1 0 0 , 0 0 \%}$ & $\mathbf{4 8 , 4 3 \%}$ \\
\hline
\end{tabular}

Fonte: Elaborado pelos autores

No conjunto das instituições financeiras brasileiras mais eficientes, levando-se em conta o porte (tabela 4), as de grande porte se destacam, apresentando um escore DEA médio de $62,15 \%$. O pior resultado foi das instituições de pequeno porte, que apresentaram um escore DEA médio de $34,37 \%$.

Este estudo revela resultados contrastantes em comparação com o de Staub; Silva e Souza e Tabak (2010), no qual as instituições financeiras de porte micro foram as mais eficientes no período estudado. Este fato pode ajudar a explicar a onda de fusões e aquisições que ocorreu no mercado financeiro, de 2000 a 2007, quando as instituições financeiras de porte grande adquiriram várias instituições de porte micro, levando em conta, dentre outros fatores possíveis, seu grau de eficiência.

Tabela 4 - Instituições financeiras mais eficientes: análise por porte

\begin{tabular}{lccccc}
\hline Porte & Eficientes & \% do Total & Amostra & \% do Total & Escore DEA Médio \\
\hline Micro & 6 & $27,27 \%$ & 20 & $20,21 \%$ & $45,63 \%$ \\
\hline Pequeno & 2 & $9,09 \%$ & 21 & $21,21 \%$ & $34,37 \%$ \\
\hline Médio & 3 & $13,64 \%$ & 29 & $29,29 \%$ & $45,59 \%$ \\
\hline Grande & 11 & $50,00 \%$ & 29 & $29,29 \%$ & $62,15 \%$ \\
\hline Total & $\mathbf{2 2}$ & $\mathbf{1 0 0 , 0 0 \%}$ & $\mathbf{9 9}$ & $\mathbf{1 0 0 , 0 0 \%}$ & $\mathbf{4 8 , 0 9 \%}$ \\
\hline
\end{tabular}

Fonte: Elaborado pelos autores

REAd | Porto Alegre - Edição 85 - № 3 - Setembro / Dezembro 2016 - p. 336 - 359 
No conjunto das instituições financeiras brasileiras mais eficientes, levando-se em conta o rating (tabela 5), pode-se verificar, claramente, uma forte relação da classificação de risco de crédito com a eficiência, pois, predominantemente, as instituições com menor risco (AAA, AA e A) são aquelas que apresentam maior eficiência, destacando-se as que possuem o rating AAA, com um escore DEA Médio de 77,07\%.

Tabela 5 - Instituições financeiras mais eficientes: análise por rating

\begin{tabular}{lccccc}
\hline Rating & Eficientes & \% do Total & Amostra & \% do Total & Escore DEA Médio \\
\hline AAA & 6 & $40,00 \%$ & 14 & $20,00 \%$ & $77,07 \%$ \\
\hline AA & 3 & $20,00 \%$ & 14 & $20,00 \%$ & $48,12 \%$ \\
\hline A & 4 & $26,67 \%$ & 16 & $22,86 \%$ & $49,51 \%$ \\
\hline BBB & 0 & $0,00 \%$ & 7 & $10,00 \%$ & $23,99 \%$ \\
\hline BB & 0 & $0,00 \%$ & 4 & $5,71 \%$ & $24,03 \%$ \\
\hline B & 2 & $13,33 \%$ & 13 & $18,57 \%$ & $39,80 \%$ \\
\hline CCC & 0 & $0,00 \%$ & 2 & $2,86 \%$ & $5,63 \%$ \\
\hline Total & $\mathbf{1 5}$ & $\mathbf{1 0 0 , 0 0 \%}$ & $\mathbf{7 0}$ & $\mathbf{1 0 0 , 0 0 \%}$ & $\mathbf{4 7 , 6 8 \%}$ \\
\hline
\end{tabular}

Fonte: Elaborado pelos autores

\section{CONSIDERAÇÕES FINAIS}

Acredita-se que a elaboração deste estudo represente uma contribuição para a construção do conhecimento sobre análise da eficiência bancária, visto que, no Brasil, há uma grande carência de trabalhos que abordem tal tema. Além disso, gera uma importante contribuição para as próprias instituições financeiras, que poderão utilizar os resultados obtidos para refletir sobre suas performances. Esta afirmação é ratificada por Oral e Yolalan (1990), ao comentarem que, nas últimas décadas, as instituições financeiras estão, constantemente, buscando ferramentas de gestão para aprimorar sua performance. Deve-se ressaltar, também, que é extremamente importante avaliar o desempenho de uma organização por meio da eficiência. Em relação a esta afirmação, pode-se recorrer ao comentário de Mester (1997): a viabilidade de uma instituição financeira em um ambiente competitivo depende, em parte, de quão eficientes suas operações são.

Ao se analisar a tabela 2, pode-se observar que, de forma geral, encontrou-se certa heterogeneidade nas instituições financeiras brasileiras mais eficientes. Conforme já 
comentado, há algumas evidências de grupos com maior ou menor eficiência dentro das classificações que foram adotadas, porém, foram identificadas instituições eficientes representando quase todas as categorias, quando se leva em conta as classificações de origem do capital (P. N. com Controle Estrangeiro, Privado Nacional (PN), Público Federal, Público Estadual e P. N. com Participação Estrangeira), setor de atuação (CDC/Consumo, Produtos, Serviços e Tesouraria, Varejo, Montadora/Veículos, Câmbio, Banco de Desenvolvimento e Corporate) e porte (Grande, Médio, Pequeno e Micro). Portanto, não se pôde constatar, com uma representatividade expressiva, a hipótese de que as classificações origem do capital, setor de atuação e porte influam diretamente na eficiência das instituições financeiras.

Tal heterogeneidade só não foi observada de forma mais acentuada quando se observa o rating de crédito, pois a imensa maioria das instituições financeiras eficientes possuem classificações AAA, AA ou A, significando que possuem uma chance muito menor de se tornarem insolventes. Acredita-se que esta seja outra contribuição do estudo, pois foram identificados indícios de que as instituições com maior solvência são, de forma comparativa, mais eficientes que as demais. Portanto, considera-se que a hipótese de que a classificação rating de crédito influa na eficiência das instituições financeiras brasileiras foi constatada.

Por outro lado, esta é uma limitação do estudo, pois, de um total de 99 instituições utilizadas na amostra, 29 não possuíam rating de crédito no sistema Visionarium, que foi a fonte utilizada para se obter tal classificação. Logicamente, caso todas as instituições tivessem o rating de crédito, a hipótese da relação diretamente proporcional entre solvência e eficiência poderia ser ratificada de forma mais consistente, ou mesmo refutada.

Sugere-se, como tema para trabalhos futuros, a utilização das duas outras principais abordagens para se avaliar a eficiência das instituições financeiras: produção e rentabilidade. Além disso, também seria importante a elaboração de estudos que levassem em conta vários anos, com o objetivo de verificar se, ao longo de um período de tempo, o grau de eficiência evidenciado se sustenta.

\section{REFERÊNCIAS}

ANDRADE, M. M. Como preparar trabalhos para cursos de pós-graduação: noções práticas. 6. ed. São Paulo: Atlas, 2004. 165 p.

ASSAF NETO, A. Mercado Financeiro. São Paulo: Atlas, 2005.

AVKIRAN, N. K. Association of DEA super-efficiency estimates with financial ratios:

Investigating the case for Chinese banks. Omega, Amsterdam, v. 39, n. 3, p. 323-334, 2011. 
BANCO CENTRAL DO BRASIL. Informações cadastrais e sobre Contabilidade. 2014. Disponível em: 〈http://www.bcb.gov.br/?infcadastro>.

BANKER, R. D.; CHARNES, A.; COOPER, W. W. Some Models for Estimating Technical and Scale Inefficiencies in Data Envelopment Analysis. Management Science, [s.1.], v. 30, n. 9, p. 1078-1092, 1984.

BERGER, A. N.; HUMPHREY, D. B. Efficiency of financial institutions: International survey and directions for future research. European Journal of Operational Research, Amsterdam, v. 98, n. 2, p. 175-212, 1997.

CARNEIRO, M. Administração de organizações: teoria e lições práticas. São Paulo: Atlas, 2012.

CHARNES, A.; COOPER, W. W.; RHODES. Measuring the Efficiency of Decision Making Units. European Journal of Operational Research, Amsterdam, v. 2, n. 6, p. 429-444, 1978.

COOPER, W. W.; SEIFORD, L. M.; ZHU, J. Data envelopment analysis history, models and interpretations. New York: Springer, 2011.

FALSARELLA JUNIOR, E. Avaliação das técnicas de fronteira na mensuração da eficiência em bancos: uma meta-análise. 2014. 194 f. Dissertação (Mestrado em PPGAO - Programa de Pós-Graduação em Administração) - Universidade de São Paulo, Ribeirão Preto, 2014.

FERREIRA, C. M. C.; GOMES, A. P. Introdução à análise envoltória de dados: teoria, modelos e aplicações. Viçosa: UFV, 2012.

FERRIER, G. D.; LOVELL, C. A. K. Measuring cost efficiency in banking: Econometric and Linear Programming Evidence. Journal of Econometrics, Amsterdam, v. 46, n. 1-2, p. 229245, 1990.

FETHI, M. D.; PASIOURAS, F. Assessing bank efficiency and performance with operational research and artificial intelligence techniques: A survey. European Journal of Operational Research, Amsterdam, v. 204, n. 2, p. 189-198, 2010.

FORTUNA, E. Mercado financeiro: produtos e serviços. 12. ed. Rio de Janeiro: Qualitymark, 1999.

HASAN, M. Z.; KAMIL, A.A.; MUSTAFA, A.; BATEN, M.A. A Cobb Douglas Stochastic Frontier Model on Measuring Domestic Bank Efficiency in Malaysia. PLoS ONE, San Franscisco, v. 7, n. 8, e42215, 2012.

KUUSSAARI, H. Productive Efficiency in Finnish Local Banking During 1985-1990. Helsinki: Suomen Pankin monistuskeskus, 1993.

LAKATOS, E. M.; MARCONI, M. A. Fundamentos de metodologia científica. 3. ed. São Paulo: Atlas, 1995. 
LANGKAMP, C. Corporate Credit Risk Management. Deutsche Nationalbibliothek: Universitãt Duisburg-Essen, 2014.

LIU, S. Slacks-based efficiency measures for predicting bank performance. Expert Systems with Applications, Amsterdam, v. 36, n. 2, p. 2813-2818, 2009.

Measuring and categorizing technical efficiency and productivity change of commercial banks in Taiwan. Expert Systems with Applications, Amsterdam, v. 37, n. 4, p. 2783-2789, 2010.

MACORIS, L. S; SALGADO JÚNIOR, A. P; FALSARELLA JÚNIOR, E. The different approaches of banking efficiency: a Meta-Analysis. In: INTERNATIONAL CONFERENCE ON DATA ENVELOPMENT ANALYSIS, 13., 2015, Braunschweig, Germany. Anais... Braunschweig: DEA, 2015.

MAXIMIANO, A. C. A. Introdução à administração. 8. ed. São Paulo, Atlas, 2011.

MESTER, L. J. Measuring efficiency at U.S. banks: Accounting for heterogeneity is important. European Journal of Operational Research, Amsterdam, v. 98, n. 2, p. 230-242, 1997.

ORAL, M.; YOLALAN, R. An empirical study on measuring operating efficiency and profitability of bank branches. European Journal of Operational Research, Amsterdam, v. 46, n. 3, p. 282-294, 1990.

PORTUGAL, M. Pronunciamentos. 2011. Disponível em:

<http://www.febraban.org.br/Noticias1.asp?id_texto=1156>. Acesso em: 07 abr. 2015.

RUDGE, L. F.; CAVALCANTE, F. Mercado de Capitais. Belo Horizonte: CNBV, 1993.

SALOMON, D. V. Como fazer uma monografia. 10. ed. São Paulo: Martins Fontes, 2001.

SATHYE, M. Efficiency of banks in a developing economy: The case of India. European Journal of Operational Research, Amsterdam, v. 148, n. 3, p. 662-671, 2003.

SEALEY, C.; LINDLEY, J. Inputs, outputs and a theory of production and cost of depository financial institutions. Journal of Finance, Hoboken, v. 32, n. 4, p. 1251-1266, 1977.

SENRA, L. F. A. C.; NANCI, L. C.; MELLO, J. C. C. B. S.; MEZA, L. A. Estudo sobre método de seleção de variáveis em DEA. Pesquisa Operacional, São José dos Campos, v. 27, n. 2, p. 191-207, maio/ago. 2007.

STAUB, R. B.; SILVA E SOUZA, G.; TABAK, B. M. Evolution of bank efficiency in Brazil: A DEA approach. European Journal of Operational Research, Amsterdam, v. 202, n. 1, p. 204-213, 2010.

TAVARES, G. A Bibliography of Data Envelopment Analysis. Piscataway, NJ: Rutcor Research Report. 2002. 
THANASSOULIS, E. Introduction to the theory and application of data envelopment analysis: a foundation text with integrated software. New York: Springer, 2003.

TORTOSA-AUSINA, E. Exploring efficiency differences over time in the Spanish banking industry. European Journal of Operational Research, Amsterdam,v. 139, n. 3, p. 643-664, 2002.

TULKENS, H.; VANDEN EECKAUT, P. How to measure efficiency and productivity with special reference to banking. Draft Report. Louvain-la-Neuve: Université Catholique de Louvain, 1995.

YANG, Z. Bank Branch Operating Efficiency: A DEA Approach. INTERNATIONAL MULTI CONFERENCE OF ENGINEERS AND COMPUTER SCIENTISTS, 2., 2009, Hong Kong. Proceedings... Hong Kong: IMECS, 2009.

YIN, R. K. Estudo de caso: planejamento e métodos. 3. ed. Porto Alegre: Bookman, 2004. 